\title{
Correlated Emission Laser and Quenching of Spontaneous Emission
}

\author{
Aditya Dahal \\ Department of Physics, Research Scholar, CMJ University, Meghalaya
}

\begin{abstract}
In this paper, it is try to show that the quantum noise leading to the laser line width can be suppressed below the standard limit (Schalow-Townes limit) by preparing the atomic systems in a coherent superposition of states as in the Hanle -Effect and Quantum Beat experiments. In such coherently prepared atoms the spontaneously emission is said to be correlated.
\end{abstract}

Keywords: Stimulated emission, Line width, Quantum noise, Quantum beat, Coherent and Spontaneous emission

\section{Introduction}

At the very outset we would like to emphasize that spontaneous emission is a universal phenomenon. The origin of the spontaneous emission can be understood only with the help of Weisskopf - Wigner theory [1] which implies that the spontaneously emitted radiation is not perfectly monochromatic but instead has a frequency spectrum with width inversely proportional to the lifetime $\tau$.

The mechanism of spontaneous emission can be understood from the quantum theory of radiation [2]. It is an isotropic perturbation always present and attributed in connection with the quantum theory of radiation to the all pervading zero - point fluctuation of the electromagnetic field. The light excites the atoms : the zero -point fluctuations de-excite them resulting in re-radiation of light. It may be noted that zero- point energy or vacuum fluctuation is the consequence of the quantization of the radiation field. With reference to laser (or stimulated emission ) it is worthwhile to say that spontaneous emission is always present in the lasing wavelength as noise, even as the wheel follows the hoof of the draught - ox, or like ones shadow that never leaves. In other words petrol or diesel drives an engine and similarly the spontaneous emission drives the stimulated emission. Now the question arises whether it is possible to remove the spontaneous noise so as to observe the pure spectral line free from spontaneous noise? The answer is not easy. But yes. A simple pictorial model for the origin of the laser linewidth envisions it as being due to the random phase diffusion process arising from the addition of spontaneously emitted photons with random phases to the laser field. The quantum noise leading to the ,laser linewidth can be suppressed below the standard Schalow - Townes limit by preparing the atomic systems in a coherent superposition of states as in the Hanle effect [3] and quantum beat experiments [4 -7]. In such coherently prepared atoms the spontaneous emission is said to be correlated. Lasers operating via such a phase coherent atomic ensemble are known a correlated emission lasers (CEL). An interesting aspect of the correlated emission lasers is that it is possible to eliminate the spontaneous emission quantum noise in the linewidths by correlating the two spontaneous emission noise events.

\section{Theory and Concept}

In active laser interferometer experiments, the limiting source of quantum noise is often spontaneous emission fluctuations in the relative phase angle. The diffusion of the relative phase angle between two such laser modes may be eliminated by preparing a laser medium consisting of three level atoms and arranging that the two transitions a $>\rightarrow \mid \mathrm{c}>$ and $|\mathrm{b}>\rightarrow| \mathrm{c}>$ drive a doubly resonant cavity as shown as in Fig1. In this way the optical paths may be differently affected by an external influence. [e .g. gravity wave ].

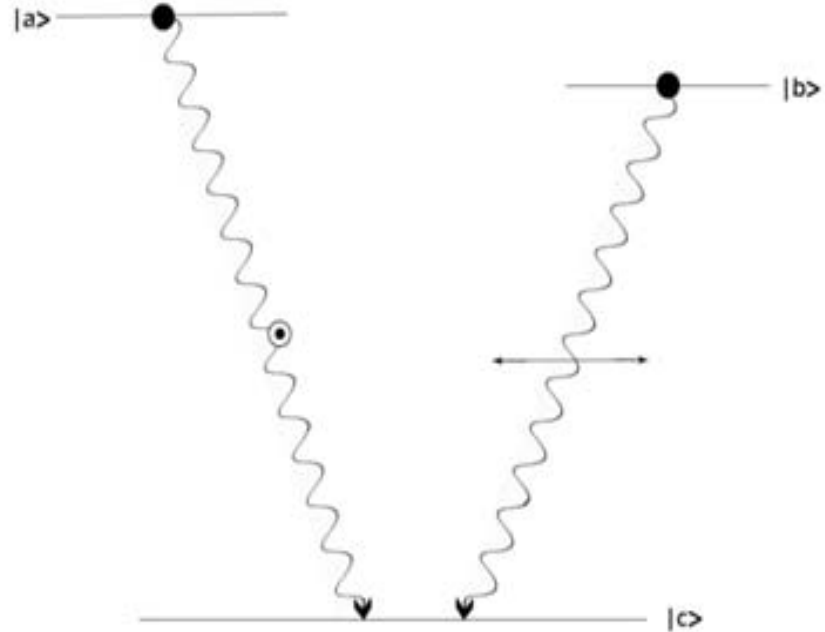

Figure 1: Hanle laser: The atoms are coherently pumped into the lasing levels $\mid \mathrm{a}>$ and $\mid \mathrm{b}>$ through an appropriately polarized pump beam. Transition from these states to the common ground state $\mid c>$ differ in their polarization.

The atomic transitions driving the two optical paths are strongly correlated when the upper levels $\mid \mathrm{a}>$ and $\mid \mathrm{b}>$ are prepared in a coherently superposition as in Hanle effect or quantum beat experiments. In the Hanle effect example, the levels $\mid a>$ and $\mid b>$ can be taken to be 'linear polarization' states formed from a single elliptical polarization state as shown in Fig 1. In the quantum beat case the coherent mixing is produced by a strong external microwave signal as shown in Fig 2(a). The fields emitted by the atoms in Fig 1 will differ in polarization while the fields produced by the atoms in Fig .2(b ) will differ in frequency. In both cases the heterodyne beat notes between 


\section{International Journal of Science and Research (IJSR) \\ ISSN (Online): 2319-7064}

Index Copernicus Value (2013): 6.14 | Impact Factor (2014): 5.611

the spontaneously emitted fields 1 and 2 shows that they are strongly correlated.

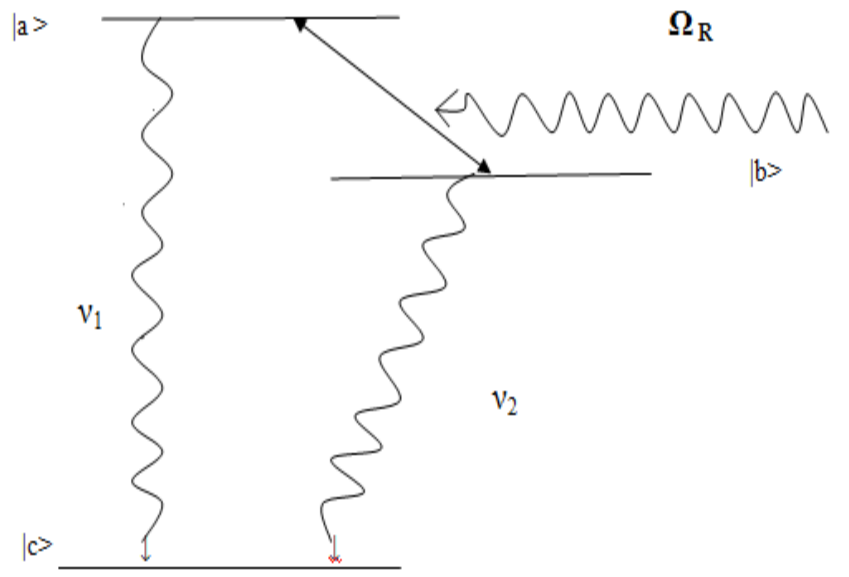

(a)

Figure 2: (a) The atoms are prepared in a coherent superposition of upper levels $\mid a>$ and $\mid b>$ by an external field with an effective Rabi frequency $\Omega_{R}$. The two laser transitions at frequencies $\mathrm{v}_{1}$ and $\mathrm{v}_{2}$ share a common lower level $\mid c>$.(b) Scheme of the quantum beat laser with doubly resonant cavity.

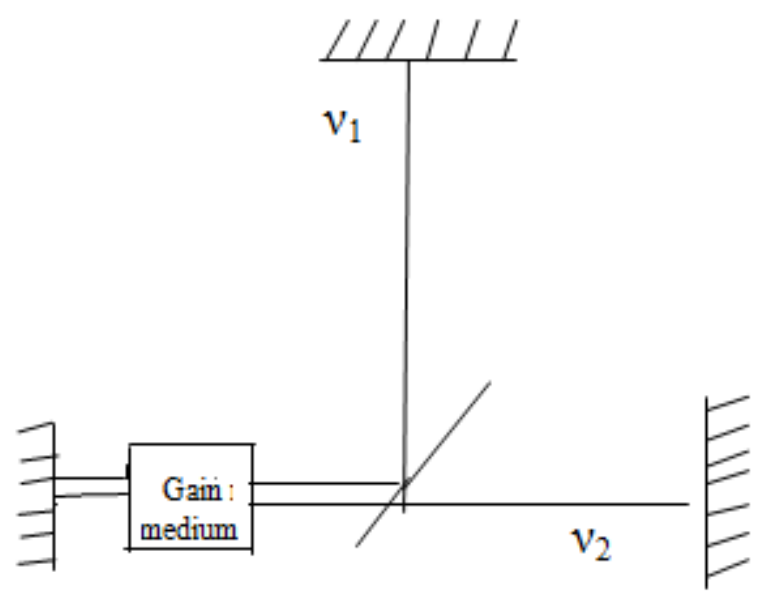

Without going into the mathematical details we can understand the quenching of the spontaneous emission fluctuations in the relative by phase $\theta$ by referring to Fig 3. Here we consider the random walk of the tips of the electric field phases of the two modes in the complex $\alpha$ - plane. If we ignore amplitude fluctuations, the phase fluctuations associated with the spontaneous emission allow the tips of the fields to diffuse out around a circle in the complex plane .When the so called diffusion co-efficient $\mathrm{D}_{\ominus \ominus}=0$ the spontaneous emission in the modes become highly correlated so that the relative phase angle is $\theta$ locked to a particular value. The average phase variables has, however, nonvanishing diffusion.

Like the Hanle effect a quantum beat laser consists of three level atoms in the $\mathrm{V}$ - configuration which are pumped in the upper level $\mid a>$ inside a doubly resonant cavity. A coherence is introduced between the upper levels $\mid a>$ and $\mid \mathrm{b}>$ by an external field which is characterized by the Rabi frequency $\Omega_{\mathrm{R}} \exp (-\mathrm{i} \varphi)$ where $\Omega_{R}$ and $\varphi$ are the real amplitude and phase. The transition $|\mathrm{a}>\rightarrow| \mathrm{c}>$ and $|\mathrm{b}>\rightarrow| \mathrm{c}>$ are assumed dipole -allowed. The transition $|a>\rightarrow| b>$ is therefore dipole forbidden. The external field leading to coherence between the levels could be a strong magnetic field for a magnetic dipole allowed transitions.

Figure 2(b): Doubly Resonant Cavity.

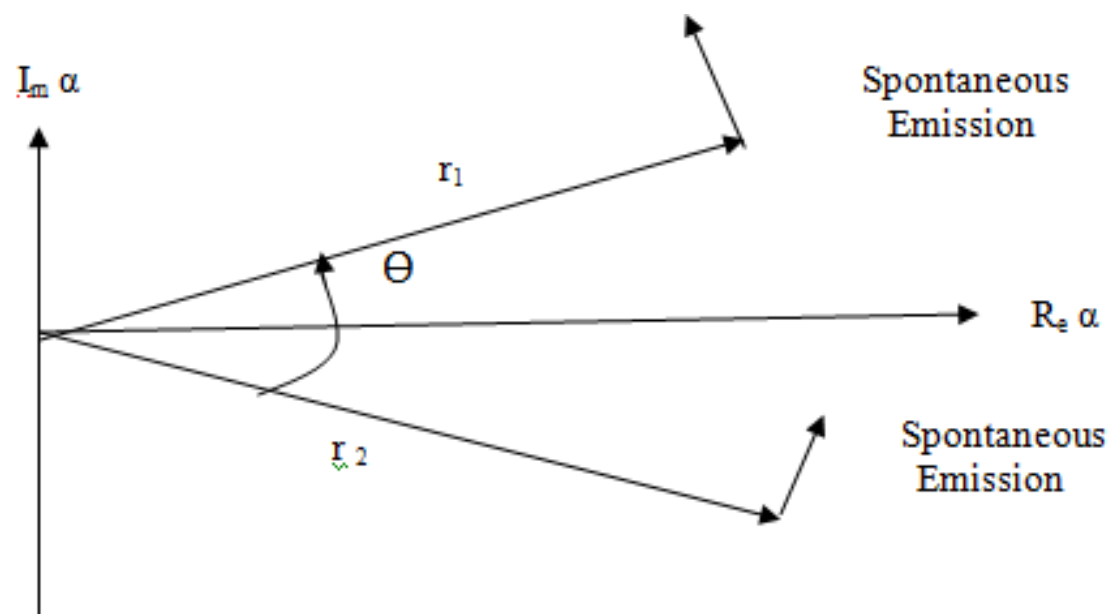

Figure 3: Pictorial representation of the correlated emission effect. The spontaneous emission events in the two modes are highly correlated and the relative phase remains the same. 


\section{Conclusions}

In conclusion, we like to state that complete quenching of spontaneous emission is ideally not possible, but it is possible to achieve this goal for a short period of time. As in the case of a coupled pendulum where the amplitude goes down decreasing but it is increasing again.

\section{References}

[1] V. F. Weisskopf and E. Wigner, Z Physik 39, 54 (1930).

[2] M. Sargent III, M.O.Scully and W.E.Lamb Jr. Laser Physics (Addition-Wesley Publishing Company, Reading, Massachusetts ) 1974.

[3] W. Hanle, Z Physics 30 ,93 (1924).

[4] M.O. Scully, H. Fearn and B.W. Atherton, in New Frontiers in Quantum Electrodynamics and Quantum optics, ed. A.O. Barut(Plenum New York 1989 )

[5] W.S. Bickel and S. Baskin, Phys. Rev. 162, 12 (1967)

[6] W.W. Chow, M.O. Scully and J.O. Stoner Jr. Phys. Rev. A $\underline{11}, 1380(1975)$

[7]M.O. Scully and J. Druhl, Phys. Rev. A, 25, 2208 (1982) 